\title{
Topical therapy of hyperendemic trachoma with rifampicin, oxytetracycline, or spiramycin eye ointments
}

\author{
S. DAROUGAR, ${ }^{1}$ B. R. JONES, ${ }^{1}$ N. VISWALINGAM,${ }^{1}$ J. ALLAMI, ${ }^{2}$ \\ D. MINASSIAN, ${ }^{1}$ M. A. FARAHMANDIAN,${ }^{3}$ AND A. HOUSHMAND ${ }^{2}$ \\ From the ${ }^{1}$ Department of Clinical Ophthalmology, Institute of Ophthalmology, Judd Street, London, \\ the ${ }^{2}$ School of Public Health, Teheran University, Iran, and the \\ ${ }^{3}$ Department of Health and Welfare, Bandar-Abbas, Iran
}

SUMmARY A double-blind stratified treatment trial was carried out on 108 patients with moderate to severe trachoma in a village in Southern Iran to assess the efficacy of topical therapy of $1 \%$ rifampicin eye ointment or $1 \%$ spiramycin eye ointment in comparison with $1 \%$ oxytetracycline eye ointment. A 6-week course of treatment with the antibiotics used twice daily produced a cure rate of $67 \%$ for rifampicin, $56 \%$ for oxytetracycline, and $64 \%$ for spiramycin when the upper tarsal conjunctiva was examined 1 week after completion of treatment. Seven months later the number of patients still free from active trachoma was reduced considerably. When the whole conjunctiva was examined, the cure rate for all 3 antibiotics was considerably lower than that obtained by examining the upper tarsus alone. The marked difference in response to treatment between the upper tarsus and the whole conjunctiva was due mainly to the presence of residual mild trachomatous inflammation in the upper fornix and the lower lid conjunctiva. The follow-up investigations revealed that this residual inflammation in the upper fornix and the lower lid is related to an increased risk of recurrence of inflammation in the whole conjunctiva, including the upper tarsal area. Microbiologically, after treatment with the antibiotics the isolation rate fell from $18-19 \%$ to $2-4 \%$. Seven months later the isolation rate in the group treated with rifampicin or oxytetracycline remained low, whereas in the group treated with spiramycin this was raised to a level of $27 \%$.

The result of this study indicates that under field conditions topical chemotherapy with rifampicin or oxytetracycline eye ointment twice daily for 6 weeks, though suboptimal, is beneficial in reducing the intensity of trachomatous inflammation and suppressing its transmission.

Hyperendemic trachoma continues to be a major cause of blindness in the rural populations of developing countries, especially those in Africa and Asia. Improvement in the standard of living and the level of rural public health would appear to be the ideal way to reduce and ultimately eradicate communicable ophthalmia, namely, trachoma and associated bacterial conjunctivitis (Jones, 1975). However, implementation of these measures is slow, expensive, and difficult and takes too long to be of considerable benefit to the present generation in danger of blindness due to these infections. Chemo-

Correspondence to Dr S. Darougar, Institute of Ophthalmology, Judd Street, London WC1H 9QS. therapy can be implemented rapidly and demands less resources. It is therefore used as an initial measure to prevent blindness in these countries.

The purpose of the present study was to assess in a double-blind stratified trial the efficacy of topical therapy of hyperendemic trachoma with rifampicin or spiramycin eye ointments in comparison with oxytetracycline eye ointment in a village in Southern Iran.

\section{Subjects and methods}

SELECTION OF PATIENTS

The child population of Tazian Pacen, a village in the Bandar-Abbas area, Southern Iran, was ex- 
amined for signs of active trachoma. Children with moderate to severe grades of trachomatous conjunctival inflammation (Dawson et al., 1975b) were included in this study.

\section{CLINICAL EXAMINATION}

Clinical signs observed with a Haag-Streit 900 slit-lamp were recorded on proformas by an extension of the scoring system used for the study of trachoma (Dawson et al., 1975b). The clinical signs observed in different areas of the palpebral conjunctiva, namely upper tarsus (UT), upper fornix (UF), lower tarsus (LT), and lower fornix (LF), were graded on a 0-3 scale and recorded for each area separately. The intensity of inflammation in the upper tarsal area or the whole conjunctiva (WC) was calculated by a modified version of the system proposed by Dawson et al. (1975b) (Table 1).

\section{PHOTOGRAPHIC DOCUMENTATION}

Each area of the palpebral conjunctiva, namely, UT, UF, LT, and LF, and the limbus of each eye were photographed with a camera unit designed for this purpose (Jones and Sheen, in preparation) and Kodachrome II film.

The colour transparencies were subsequently examined in London, the clinical signs being quantified by 3 ophthalmologists following the method used during clinical examination. In case of disagreement among the observers transparencies were re-examined and compared with a standard set.

\section{LABORATORY INVESTIGATIONS}

Conjunctival swabbings collected from the upper tarsus, upper fornix, and the lower lid (LL) conjunctiva of the right and left eyes were placed together in a plastic capsule containing 2SP transport mediun (Gordon et al., 1969) with additional $3 \%$ fetal calf serum and stored in a liquid nitrogen refrigerator $\left(-180^{\circ} \mathrm{C}\right)$ until transported to the

Table 1 Grades of intensity of active trachoma

\begin{tabular}{lllll}
\hline & & Papillae & \multicolumn{2}{l}{ Follicle score } \\
\cline { 5 - 5 } & Intensity & score & Grade & Type* \\
\hline 0 & No trachoma & $0-3$ & 0 & 0 \\
1 & $\begin{array}{c}\text { Insignificant } \\
\text { or inactive }\end{array}$ & $0-3$ & $1-3$ & 4 \\
2 & Mild & $1-2$ & $1-2$ & $1-3$ \\
3 & Moderate & $1-2$ & 3 & $1-3$ \\
4 & Severe & 3 & $1-3$ & $1-3$ \\
\hline
\end{tabular}

- Type of follicles: $0=$ no follicles, $1=$ follicles $<0.5 \mathrm{~mm}$ in diameter, $2=$ follicles between 0.5 and $1 \mathrm{~mm}$ in diameter, $3=$ follicles $>1 \mathrm{~mm}$ in diameter, $4=$ old follicles. laboratory in London. Here they were stored at $-70^{\circ} \mathrm{C}$ until the time of inoculation. The methods of culture in irradiated McCoy cells and the identification of the isolates have been described elsewhere (Darougar et al., 1971).

\section{RANDOM ALLOCATION}

Children with active trachoma were divided into 3 groups according to a randomisation schedule stratified for age, sex, intensity of inflammation, and the number of children with active trachoma in each family.

\section{MEDICATION}

Each patient was treated with one of the 3 antibiotics, namely, rifampicin $1 \%$ eye ointment (supplied by Lepetit and Co. Ltd.), spiramycin (Rovamycin) $1 \%$ eye ointment (supplied by May and Baker Ltd.), or oxytetracycline $1 \%$ eye ointment (supplied by Pfizer Ltd.). The antibiotics were applied by experienced field technicians to the conjunctival sac twice daily for a period of 6 weeks. Those who received a total of less than 70 applications were excluded from this study. Of the patients included in the trial all except 2 received the maximum number of applications of ointment.

\section{FOLLOW-UP INVESTIGATIONS}

After initial clinical and laboratory examinations treatment was started in April 1974. The follow-up examinations with the collections of specimens were made at 1 week, 4 months, and 7 months after completion of treatment.

\section{STATISTICAL ANALYSIS}

Chi-square significance tests were used for a comparison of results.

\section{Results}

Initially 148 patients with moderate to severe trachoma (grades 3 or 4 ) in their whole conjunctiva were included in this trial. Of these, 40 were excluded, 22 due to inadequate treatment or follow-up and 18 because of the development of an adverse reaction to spiramycin. The allocation of the 108 remaining patients to the 3 treatment groups and their age and sex distribution are shown in Table 2.

Because of the close correlation observed between the results of screening transparencies and the clinical examination, and the high repeatability of scoring inflammatory changes by photography, the efficacy of treatment in this trial was assessed by scoring coded transparencies taken before and at intervals after the treatment.

When the upper tarsus alone was examined (the 
Table 2 Allocation of patients to various treatments, and their age and sex distribution

\begin{tabular}{lllllll}
\hline Treatment & $\begin{array}{l}\text { No. of } \\
\text { patients }\end{array}$ & $0-1$ & $2-4$ & $5-9$ & $10-14$ & $\begin{array}{l}\text { Sex } \\
M / F\end{array}$ \\
\cline { 2 - 7 } & 45 & $4(9 \%)$ & $22(49 \%)$ & $17(38 \%)$ & $2(4 \%)$ & $60 / 40$ \\
Rifampicin & 41 & $4(10 \%)$ & $22(54 \%)$ & $14(34 \%)$ & $1(2 \%)$ & $54 / 46$ \\
Oxytetracycline & $22 *$ & $6(27 \%)$ & $11(50 \%)$ & $5(23 \%)$ & 0 & $41 / 59$
\end{tabular}

*Eighteen patients were excluded from the trial because of the development of an adverse reaction to spiramycin.

conventional method) 1 week after completion of treatment, the cure rate for rifampicin was $67 \%$ compared with $56 \%$ for oxytetracycline and $64 \%$ for spiramycin (Table 3 and Fig. 1). The beneficial effects of treatment with each of the 3 antibiotics were statistically significant $(P<0.05)$. The differences between the efficacy of these antibiotics were not statistically significant. At the follow-up examination 7 months later the number of patients who were still free from active trachoma was reduced to $36 \%$ for rifampicin compared with $32 \%$ for oxytetracycline and $27 \%$ for spiramycin (Table 3 and Fig. 1).

When the trachomatous inflammatory changes were assessed in the whole conjunctiva, the cure rate for all 3 antibiotics was considerably lower than that obtained by examining the upper tarsus alone (Table 4 and Fig. 2). When the whole conjunctiva was examined 1 week after completion of treatment, the cure rate for rifampicin was $38 \%$ compared with $27 \%$ for oxytetracycline and $18 \%$
Table 3 Number of patients cured examining the upper tarsal conjunctiva alone at various times after treatment

\begin{tabular}{lllll}
\hline \multirow{2}{*}{$\begin{array}{l}\text { Type of } \\
\text { treatment }\end{array}$} & $\begin{array}{l}\text { No. of } \\
\text { patients }\end{array}$ & \multicolumn{2}{l}{ No. cured } & \\
\cline { 4 - 5 } & 1 week & 4 months & 7 months \\
\hline Rifampicin & 45 & $30(67 \%)$ & $22(49 \%)$ & $16(36 \%)$ \\
Oxytetracycline & 41 & $23(56 \%)$ & $15(37 \%)$ & $13(32 \%)$ \\
Spiramycin & 22 & $14(64 \%)$ & $8(36 \%)$ & $6(27 \%)$ \\
\hline
\end{tabular}

for spiramycin (Table 4 and Fig. 2). In the second follow-up 4 months later the number of patients still free from trachoma in the rifampicin group $(40 \%)$ was higher than those treated with oxytetracycline $(20 \%)$ or spiramycin $(14 \%)$. This difference reaches statistical significance at a level of $5 \%$ $(P<0.05)$. However, this advantage of rifampicin was no longer significant at the third follow-up examination 7 months later.
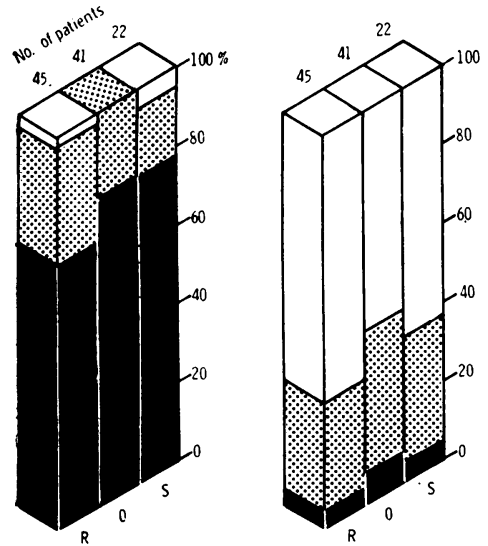

$$
\begin{aligned}
& \text { BEFORE } \\
& \text { TREATMENT }
\end{aligned}
$$

Moderate or severe trachoma

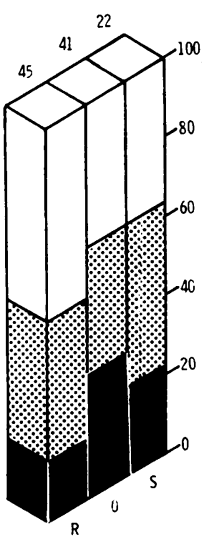

FOUR MONTHS AFTER COMPLETION
OF TREATMENT

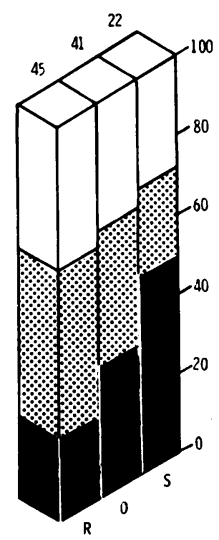

Fig. 1 Changes in the grades of intensity of trachoma in the upper tarsal conjunctiva after treatment with rifampicin $(R)$, oxytetracycline $(O)$, and spiramycin $(S)$ 
Fig. 2 Changes in the grades of intensity of trachoma in the whole conjunctiva after treatment with rifampicin $(R)$, oxytetracycline $(O)$, and spiramycin $(S)$

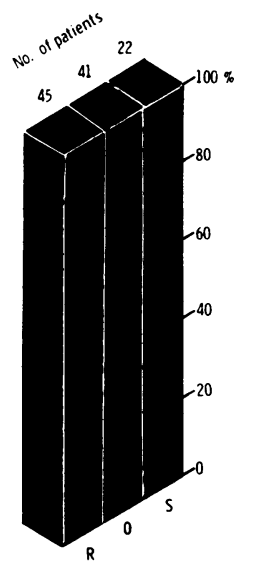

BEFORE BEFORE
TREATMENT

Moderate or severe trachoma
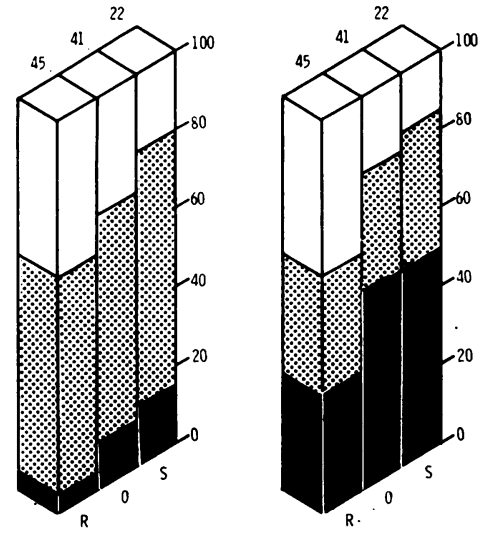

$$
\begin{aligned}
& \text { FOUR MONTHS } \\
& \text { AFTER COMPLETION } \\
& \text { OF TREATMENT }
\end{aligned}
$$

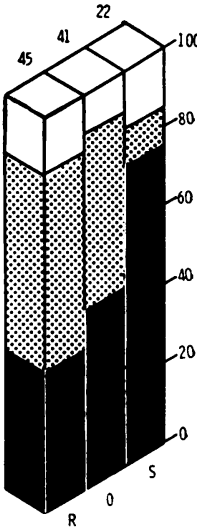

SEVEN MONTHS AFTER COMPLETION OF TREATMENT
Table 4 Number of patients cured examining the

\begin{tabular}{|c|c|c|c|c|}
\hline \multirow{2}{*}{$\begin{array}{l}\text { Type of } \\
\text { treatment }\end{array}$} & \multirow{2}{*}{$\begin{array}{l}\text { No. of } \\
\text { patients }\end{array}$} & \multicolumn{3}{|l|}{ No. cured } \\
\hline & & 1 week & 4 months & 7 months \\
\hline Rifampicin & 45 & $17(38 \%)$ & $18(40 \%)$ & $7(16 \%)$ \\
\hline Oxytetracycline & 41 & $11(27 \%)$ & $8(20 \%)$ & $4(10 \%)$ \\
\hline Spiramycin & 22 & $4(18 \%)$ & $3(14 \%)$ & $4(18 \%)$ \\
\hline
\end{tabular}
whole conjunctiva at various times after treatment

In those patients who were not cured, the intensity of inflammatory changes in the upper tarsus as well as the whole conjunctiva was markedly reduced after the treatment with the 3 antibiotics (Figs. 1 and 2). The number of patients with grades 3 and 4 fell to $4 \%$ after treatment with rifampicin compared with $12 \%$ for oxytetracycline and $14 \%$ for spiramycin (Fig. 2). In the second follow-up 4 months later the intensity of inflammation in those patients who were not cured was significantly less with rifampicin than with either of the other 2 antibiotics (Figs. 1 and 2) $(P<0.05)$. This difference was not observed during the third follow-up.

The marked difference in responses to treatment between the upper tarsus and the whole conjunctiva was mainly due to the presence of residual mild trachomatous inflammation (grade 2) in the upper fornix and lower lid conjunctiva (Figs. 1 and 2). However, the follow-up studies in all groups treated with antibiotics showed that residual mild inflammation in the upper fornix and lower lid conjunctiva is related to an increased risk of recrudescence of inflammation in the whole conjunctiva, including the upper tarsal area (Table 5).

No marked differences in response to treatment were observed between different age groups except in babies under 1 year old, who showed a poor response to treatment. There was no significant difference between males and females in response to treatment.

Microbiologically between 18 and $19 \%$ of patients allocated to each treatment group were positive for

Table 5 Number of recurrences and/or reinfections in 108 patients with or without inflammatory changes in their upper tarsus (UT) or the rest of their conjunctiva; upper fornix, and lower lid $(U F+L L)$

\begin{tabular}{|c|c|c|c|c|c|}
\hline \multirow[t]{2}{*}{ Group } & \multirow{2}{*}{$\begin{array}{l}1 \text { week after } \\
\text { treatment } \\
\text { Grade of inflammation }\end{array}$} & \multirow[t]{2}{*}{$\begin{array}{l}\text { No. of } \\
\text { patients }\end{array}$} & \multicolumn{3}{|c|}{$\begin{array}{l}7 \text { months after treatment } \\
\text { No. of recurrences/ } \\
\text { reinfections }(U T+U F+L L)\end{array}$} \\
\hline & & & Mild & $\begin{array}{l}\text { Moderate } \\
\text { severe }\end{array}$ & Total \\
\hline 1 & $\left\{\begin{array}{l}\mathbf{U T}=\text { inactive } \\
\mathbf{U F}+\mathbf{L} \mathbf{L}=\text { inactive }\end{array}\right.$ & 32 & 12 & $12 *$ & $24(75 \%)$ \\
\hline 2 & $\left\{\begin{array}{l}\mathbf{U T}=\text { inactive***} \\
\mathbf{U F}+\mathbf{L I}=\text { mild to } \\
\text { severe }\end{array}\right.$ & 35 & 7 & $25 *$ & $32(91 \%)$ \\
\hline 3 & $\left\{\begin{array}{l}\mathrm{UT}=\text { mild to } \\
\text { severe*** } \\
\mathrm{UF}+\mathrm{LL}=\text { mild to } \\
\text { severe }\end{array}\right.$ & 41 & 16 & $21 *$ & $37(90 \%)$ \\
\hline
\end{tabular}
after treatment with antibiotics

-The difference between group 1 and groups 2 and 3 combined is statistically significant at a level of $5 \%(P<0.05)$. *Figs. 1 and 2 . 
Table 6 Number of positive cultures for C. trachomatis before and after chemotherapy

\begin{tabular}{llllll}
\hline \multirow{2}{*}{$\begin{array}{l}\text { Type of } \\
\text { treatment }\end{array}$} & $\begin{array}{l}\text { No. of } \\
\text { patients }\end{array}$ & \begin{tabular}{l} 
No. of positives \\
\cline { 3 - 6 }
\end{tabular} & & $\begin{array}{l}\text { Before } \\
\text { treatment }\end{array}$ & After treatment \\
\cline { 3 - 6 } & & & 1 week & 4 months & 7 months \\
\hline Rifampicin & 45 & $8(18 \%)$ & $2(4 \%)$ & 0 & $1(2 \%)$ \\
Oxytetracycline & 41 & $8(19 \%)$ & $1(2 \%)$ & $2(5 \%)$ & 0 \\
Spiramycin & 22 & $4(18 \%)$ & $1(4 \%)$ & $1(4 \%)$ & $6(27 \%)$ \\
\hline
\end{tabular}

Chlamydia trachomatis in irradiated McCoy cell culture (Table 6). Follow-up tests 1 week and 4 months after completion of treatment showed a considerable reduction in the number of positives in all 3 groups (Table 6). However, in the last follow-up 7 months later the isolation rate for the group treated with spiramycin was increased to a level of $27 \%$, but no significant changes were observed in the positivity rates for those patients who were treated with rifampicin or oxytetracycline. This difference is significant $(P<0.005)$.

\section{ADVERSE REACTIONS}

Of 40 patients who were treated with spiramycin eye ointment 18 developed an adverse reaction 1-3 weeks after the start of their treatment. This consisted of watering, moderate discharge, moderate to severe swelling of lids associated with erythema, together with oedema and increased hyperaemia of the bulbar and palpebral conjunctiva. These symptoms and signs were increased with further applications of the ointment. However, after treatment was stopped the reaction subsided gradually in about a week.

Apart from slight discomfort and irritation, no other reaction was obser ied after applications of rifampicin or oxytetracycline eye ointments.

\section{Discussion}

In this trial preschool children with moderate to severe trachoma were included. Originally stratified randomisation for age, sex, grades of intensity of trachoma, and number of children with active trachoma in the family of patients produced a wellbalanced distribution of patients in the 3 different treatment groups. However, because of the development of adverse reactions in patients treated with spiramycin eye ointment the distribution of patients in this group became somewhat different from the other 2 groups (Table 2).

The treatment trial was designed to test the efficacy of different topical drugs on hyperendemic trachoma under field conditions. The eye ointments were applied twice daily. This schedule is more practical than thrice daily applications of ointment in the rural areas of Iran, where during the day adults, mostly accompanied by their young children, are working on their farms. During the 6-week course of treatment the total number of ointments applied was 84 .

Examination of the upper tarsus alone 1 week after the completion of treatment showed a moderate cure rate for rifampicin, oxytetracycline, and spiramcyin (56\% to $67 \%$ ). The intensity of disease was also reduced considerably in patients who were not cured. At the follow-up examination 7 months later the number of patients free from trachoma in all groups was halved (27\% to $36 \%)$ and the intensity of disease increased markedly.

When the inflammatory changes of trachoma in the whole conjunctiva were assessed, the cure rate for all 3 antibiotics was markedly lower than that obtained by examining the upper tarsal conjunctiva alone. This difference was due to the presence of active but mild to moderate trachoma in the fornices of some of the patients with normal upper tarsal conjunctiva. In this group of patients (with normal upper tarsal conjunctiva but with active trachoma in their fornices) the rate of recurrence or recrudescence of moderate to severe trachoma was twice as high as in those who showed no active trachomatous inflammation in their whole conjunctiva, including the upper tarsus (Table 5).

In Tunisia treatment of hyperendemic trachoma with rifampicin or tetracycline ointments twice daily for a course of 10 weeks was shown to be beneficial in suppressing the disease but not in eradicating the infection (Dawson et al., 1975a).

In London treatment of paratrachoma (inclusion conjunctivitis, TRIC punctate keratoconjunctivitis and trachoma) of sexually transmitted origin (Jones, 1975) with rifampicin eye ointment 3 times daily for 6 to 7 weeks was shown to eradicate the infection in $90 \%$ of patients (Darougar et al., 1977). However, in the absence of apparent differences in the sensitivity of prototypes of TRIC agent serotypes as well as wild TRIC agent isolates to rifampicin, tetracyclines, and spiramycin (Treharne et al., 1977) it can be argued that in the present study the lower cure rate obtained with rifampicin may be due to the suboptimal daily applications of the ointment or to a shorter course of treatment.

In this trial a control group was not included to assess the degree of natural decline in the intensity of trachoma with the lapse of time, increasing age, or seasonal variations. However, studies in Tunisia have shown that in children under the age of 6-7 with moderate to severe trachoma the intensity of 
inflammatory changes in the conjunctiva remains fairly constant in a period of 1 year (Dawson et al., 1975a). In the present study the majority of the patients included were children under the age of 7 with moderate to severe trachoma, and the course of follow-up was up to 7 months. Therefore it can be argued that the beneficial effect obtained in this trial is probably caused mainly by chemotherapy and not by the natural decline in the intensity of trachoma.

Immediately after completion of treatment the efficacy of topical therapy with rifampicin was very similar to that of oxytetracycline or spiramycin. In the second follow-up (4 months later) rifampicin appeared clinically to be superior to oxytetracycline or spiramycin by having fewer recurrences. In the last follow-up (7 months later) this difference was no longer present. At this time the cure rates for all 3 antibiotics were as low as $20 \%$ (whole conjunctiva). In this study the children in the trial were living with other untreated children and adults in the village, and no attempt was made to reduce the possibility of reinfections in these children by treating their families.

Microbiologically the chlamydial isolation rate before treatment was similar in the 3 groups (18$19 \%$ ). At the last follow-up (7 months later) the isolation rate in groups treated with rifampicin or oxytetracycline remained low, whereas in the group treated with spiramycin it was increased to a level of $27 \%$. The microbiological response to treatment with rifampicin or oxytetracycline obtained in this study is similar to those reported in trials in Tunisia (Dawson et al., 1975a) and London (Darougar et al., 1977).

In the group treated with spiramycin 18 patients developed moderate to severe adverse reactions. Bacterial cultures of samples of spiramycin ointments used in this study showed no growth. In London no adverse reaction was observed in patients with chlamydial, viral, or bacterial conjunctivitis who were treated with spiramycin eye ointment from the same batch. Although contact dermatitis due to spiramycin eye ointment has been reported previously (Hurwitz, 1959), the cause of this unusual reaction in children in Iran remains obscure.
In evaluating the effect of trachoma chemotherapy with rifampicin, oxytetracycline, or spiramycin we conclude that, though topical therapy with these antibiotics twice daily for 6 weeks is suboptimal, it may convert blinding trachoma in a community 7 into a nonblinding disease (Dawson et al., 1975b) if applied to the entire population.

The authors are grateful to the Dean of the School of Public Health, Teheran, Iran, and the Director of the $\infty$ Department of Health and Welfare, Bandar-Abbas, Iran, $\overrightarrow{0}$ for their support of this project. We are indebted to $\mathrm{Mr} \mathrm{H}$. Shah Mohamadi for his assistance in the collection of clinical specimens, and to Mr R. Hejazi for his technical assistance in the laboratory tests.

The research project was partially supported by grants from the Dulverton trust, the Wellcome Foundation, and or the Order of St John.

\section{References}

Darougar, S., Kinnison, J. R., and Jones, B. R. (1971). Simplified irradiated McCoy-cell culture for isolation of Chlamydia. In Trachoma and Related Disorders Caused by Chlamydial Agents, pp. 63-70. Edited by R. L. Nichols. Excerpta Medica: Amsterdam.

Darougar, S., Viswalingam, M., Treharne, J. D., Kinnison, J. R., and Jones, B. R. (1977). Treatment of TRIC infection of the eye with rifampicin or chloramphenicol. British Journal of Ophthalmology, 61, 255-259.

Dawson, C. R., Hoshiwara, I., Daghfous, T., Messadi, M., Vastine, D. W., and Schachter, J. (1975a). Topical tetracycline and rifampicin therapy of endemic trachoma in Tunisia. American Journal of Ophthalmology, 79, 803-811.

Dawson, C. R., Jones, B. R., and Darougar, S. (1975b). Blinding and non-blinding trachoma: assessment of intensity of upper tarsal inflammatory disease and disabling lesions. Bulletin of the World Health Organisation. 52, 279-282.

Gordon, F. B., Harper, I. A., Quan, A. L., Treharne, J. D. Dwyer, R. St. C., and Garland, J. A. (1969). Detection of Chlamydia (Bedsonia) in certain infections in man, 1 . Laboratory procedures: comparison of yolk sac and cell culture for detection and isolation. Journal of Infectious Diseases, 120, 451-462.

Hurwitz, P. (1959). Experience with spiramycin. A new antibiotic in ophthalmic infections. Antibiotic Annuals, pp. 199-201. Medical Encyclopaedia, Inc.: New York.

Jones, B. R. (1975). The prevention of blindness from trachoma. Transactions of the Ophthalmological Societies of the United Kingdom, 95, 16-33.

Treharne, J. D., Day, J., Yeo, C. K., Jones, B. R., and Squires, S. (1977). Susceptibility of Chlamydia to chemotherapeutic agents. In Nongonococcal Urethritis and Related Infections, pp. 214-222. Edited by D. Hobson and K. K. Holmes. American Society for Microbiology. 